\title{
Performance of different colour quality metrics proposed to CIE TC 1-91
}

\author{
Pramod Bhusal ${ }^{1, *}$, Rajendra Dangol ${ }^{1,2}$ \\ ${ }^{1}$ Department of Electrical Engineering and Automation, Lighting Unit, Aalto University, School of \\ Electrical Engineering, Espoo, Finland \\ ${ }^{2}$ Eindhoven University of Technology, Department of the Built Environment, Building Lighting \\ Group, Eindhoven, the Netherlands
}

Received 22 June 2017, Accepted 24 July 2017

\begin{abstract}
The main aim of the article is to find out the performance of different metrics proposed to CIE TC 1-91. Currently, six different indexes have been proposed to CIE TC 1-91: Colour Quality Scale (CQS), Feeling of Contrast Index (FCI), Memory colour rendering index (MCRI), Preference of skin (PS), Relative gamut area index (RGAI) and Illuminating Engineering society Method for evaluating light source colour rendition (IES TM-30). The evaluation and analysis are based on previously conducted experiment in lighting booth. The analysis showed the area based metric FCI was good subjective preference indicator. The subjective preference was measured in terms of naturalness of objects, colourfulness of colour checker chart, and the visual appearance of the lit scene in the booth.
\end{abstract}

Keywords: color quality, color rendering, LED light sources, colorimetry

\section{Introduction}

The lighting technology based on light emitting diodes (LEDs) has already emerged and started to enter the general lighting market taking over many lighting applications. It is well known fact that the LED based lighting systems have great potential for significant energy saving. After the invention of blue LED, LED based lighting technology has developed rapidly and is competitive alternative for general lighting. An LED luminaire with efficacy of $200 \mathrm{~lm} / \mathrm{W}$ has already been demonstrated by Cree(Cree, 2014). However, the luminous efficacy is not the only key factor that determines an extensive and successful penetration of LEDs into the general lighting. The end-user satisfaction and acceptance are equally important as the efficacy for the successful implementation of LED based lighting system. However, colour quality of light sources is still judged with flawed International Commission on Illumination (CIE) colour rendering index (CRI). Since 1993, CIE has been trying to update the CIE CRI as it has many deficiencies but it has failed to update. Many CIE technical committees (TCs) have been formed and closed after few years, as they couldn't come to general agreement between the TC members. The recently closed TC is CIE TC 1-69. In 2012, again two new TCs were formed; CIE TC 1-90 and CIE TC-91. The main aim to form two TCs is to distinguish between colour fidelity; CIE TC 1-90 and colour preference along with naturalness of object; CIE TC 1-91. The main aim of the article is to find out the performance of different metrics proposed to CIE TC 1-91. Currently, six different indexes have been proposed to CIE TC 1-91 (explained in chapter 2).

In this article, the ability of different metrics to predict the rank order of different light sources based upon the naturalness of objects, the visual appearance in the booth and the colourfulness of Macbeth colour checker chart are studied. Also, the correlation between the observers mean rating for the naturalness of objects, the visual appearance in the booth and the colourfulness of Macbeth colour checker chart with different proposed metrics are analysed.

\footnotetext{
*Bhusal P, Tel: +358503160991

Email address: Pramod.bhusal@aalto.fi
} 


\section{Various proposed metrics in CIE TC 1-91 for evaluating the colour quality of white light sources}

The terms of reference of CIE TC 1-91 (New methods for evaluating the Colour quality of white-light sources) is to evaluate available new methods for evaluating the colour quality of white-light sources with a goal of recommending methods for industrial use. Currently, TC 1-91 is evaluating following different indexes to include in the technical report:

1. Colour quality Scale (CQS)

2. Feeling of Contrast index (FCI)

3. Memory colour rendering index (MCRI)

4. Preference index of skin (PS)

5. Illuminating Engineering society Method for evaluating light source colour rendition (IES TM-30)

6. Relative Gamut area index (RGAI)

\subsection{Colour quality scale}

Colour quality scale (CQS) aims to describe several aspects of colour quality including colour rendering, chromatic discrimination and observer preferences (Davis \& Ohno, 2010). The method for calculating the CQS is derived from CIE Test Sample Method(CIE, 1995) with modifications. CQS uses 15 Munsell samples covering low to high chromatic saturation. The reference illuminant is selected as in CIE CRI. The tristimulus values of illuminated samples are corrected for chromatic adaptation by using CMCCAT2000 (Li, Luo, Rigg, \& Hunt, 2002) and CIELAB colour space is used instead of CIE $1964 \mathrm{~W}^{*} \mathrm{U} * \mathrm{~V}^{*}$ colour space. The saturation factor (Davis \& Ohno, 2010) is introduced in calculating the colour difference of each reflective sample in CQS. The saturation factor is considered to neutralize the colour difference that arises from an increase in object chroma from test source relative to the reference illuminant. To ensure the influence of large hue shifts of any sample on the results, the root-mean-square (RMS) of colour shifts of each individual sample is used on the CQS rather than arithmetic mean. CQS also includes graphical method called Colour Saturation Icon to provide more comprehensive information. Colour Saturation Icon shows the saturation of the 15 colour samples with different hues under a test SPD compared to that under a reference SPD, either more saturated or desaturated.

\subsection{Feeling of contrast index (FCI)}

Feeling of contrast index (FCI) describes quantitative degree of the feelings of contrast for the test lamp to the reference illuminant (K Hashimoto, Yano, Shimizu, \& Nayatani, 2007). FCI is based on CIELAB gamut area of four colour combination under the test light source at illuminace $1000 \mathrm{~lx}(\mathrm{G}(\mathrm{T}, \mathrm{Et}=1000 \mathrm{~lx}))$ and under a CIE Standard Illuminant D65 (as reference) at illuminace $1000 \mathrm{~lx}(\mathrm{G}(\mathrm{D} 65 ; \mathrm{Er}=1000 \mathrm{~lx}))$. The four-colour combination consists of Red (5R4/12), Blue (4.5PB3.2/6), Yellow (5Y8.2/10) and Green (5.5G5/8), and these colours are selected with the consideration that they represent almost all the hues used in the actual environment (Kenjiro Hashimoto \& Nayatani, 1994). FCI is calculated by using equation (1).

$$
F C I=\left[\frac{G\left(T, E_{t}=1000 l x\right)}{G\left(D_{65}, E_{r}=1000 l x\right)}\right]^{1.5} \times 100
$$

\subsection{Memory Colour Rendering Index}

The memory CRI (MCRI) is the colour rendering metric based on memory colours (Smet, Ryckaert, Pointer, Deconinck, \& Hanselaer, 2012, 2010; Smet, Ryckaerta, Deconinckb, \& Hanselaera, 2010). Memory colours are those colours that are associated with familiar objects in long term memory. The proposed MCRI is calculated by determining the perceived similarity between an object's apparent colour and its memory colour. Initially, MCI used nine familiar objects: a green apple, a banana, orange, dried lavender, a smurf figurine, strawberry yoghurt, a sliced cucumber, a cauliflower and Caucasian skin. Later, a neutral grey sphere was added to the set(Smet et al., 2012). The tristimulus values of these objects under D65 illuminant are transformed to the IPT colour space and corresponding similarity distribution $S_{i}(X)$ are computed. The similarity distribution is a set of special colour quality indicators that describes the degree of similarity with the object's memory colour. The test sources will have better colour rendering properties if the degree of similarity is higher. The degree of similarity with the memory colours were calculated by using the similarity distributions. Then, the general degree of memory colour similarity is obtained by taking the geometric mean of the 10 $S_{i}$. Finally, Si and Sa are rescaled to specific and general colour quality indices, $R_{m, I}$ and $R_{m}$. $R_{m}$ is the general memory colour rendering index (MCRI). 


\subsection{Preference index of skin}

Preference index of skin (PS) metric is based on preferred Japanese complexion and is considered as one of colour rendering methods of light sources. PS was developed in order to evaluate the degrees of preferred complexion quantitatively. It uses CAM02 colour appearance model. The procedure for calculating the preference index PS is shown in Fig. 1 with help of flowchart (Yano \& Hashimoto, 2015).

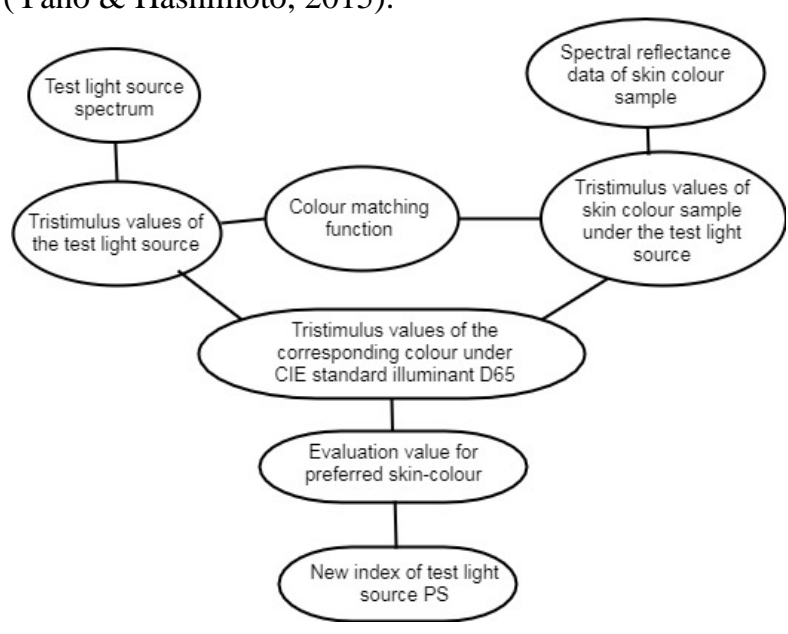

Fig. 1. Flow chart to calculate preference index (PS) (Yano \& Hashimoto, 2015)

\subsection{IES TM-30}

IES TM-30 Method for Evaluating Light Source Colour Rendition is a two-measure system that evaluate the colour rendition properties of light sources. The system measure the colour fidelity (Rf) and the colour gamut (Rg) of a light source in comparison to a reference illuminant having same CCT as that of test light source. The reference illuminant is Planckian radiator for light sources with CCT below $4500 \mathrm{~K}$, a linear combination of blackbody radiator for light sources with CCT from $4501 \mathrm{~K}$ to $5499 \mathrm{~K}$, and a CIE D series illuminant for light sources with CCT above $5500 \mathrm{~K}$. It uses 99 colour evaluation samples (CES) and all of them are from real and natural objects including paints, fabrics, plastics and skin tones. Moreover, it uses CAM02-UCS uniform colour space. Both the measures, colour fidelity (Rf) and colour gamut (Rg) share a common set of colour samples and colour space. The fidelity index(Rf) is calculated with the help of Equation (2) and rescaling to the final Rf is accomplished using Equation (3) (for more detail see (David et al., 2015; Illuminating Engineering Society (IES), 2015)).

$$
R_{f}^{\prime}=100-7.54\left(\left(\frac{1}{99}\right) \sum_{i=1}^{99}\left(\Delta E_{J a b, i}\right)\right)
$$

where,

7.54 is the scaling factor determined such that the mean CIE CRI value for the CIE F series illuminants i.e.F1 to F12 is equal to the mean $\mathrm{Rf}$ value for the same illuminants

$\Delta E_{J a b, i}$ is the colour difference between each CES under the test and reference illuminant in CAM02-UCS colour space

$$
R f=10 \ln \left(e^{R^{\prime} f} / 10+1\right)
$$

Whereas, Rg is a relative measure of the average area spanned by the 99 colour samples in the uniform colour space i.e. CAM02-UCS. Based on the chromaticity of CES under the reference illuminant, the colour coordinates (a', b') of CES under the test source and reference illuminant are grouped into 16 hue bins of equal width. For each bin, the average values of a' and b' will be calculated, resulting hexadecagon i.e. 16-sixteen sided polygon for the reference and test samples. Then, Rg is calculated using Equation (4) (for more detail see (David et al., 2015; Illuminating Engineering Society (IES), 2015))

$$
R g=100 \cdot \frac{A_{\text {test }}}{A_{\text {ref }}}
$$

where, $\mathrm{A}$ is the area of hexadecagon

TM30 also generates a colour graphic showing the average hue and saturation shifts to help with interpreting the values of Rf and Rg.

\subsection{Relative gamut area index (RGAI)}


The relative gamut area (RGAI) is calculated as the ratio of gamut area formed by the test light source to the gamut area formed by the reference illuminant defined by the chromaticities of the same eight test samples used by CIE CRI in CAM02-UCS colour space. Detailed procedure to calculate relative gamut area index can be found in the reference (Teunissen, Van Der Heijden, Phd, \& De Beer, n.d.).

\section{Experimental set-up}

The experiment was conducted in a dark room with the help of three sections lighting booth. Each section of a booth had the dimensions of $1 \mathrm{~m}$ height, $0.5 \mathrm{~m}$ depth and $0.5 \mathrm{~m}$ width. The inner surface of the booth was coated with matt grey paint (reflectance $=0.5$ ). Seven LED SPDs and one fluorescent lamp SPDs each at CCTs of $2700 \mathrm{~K}, 4000 \mathrm{~K}$ and 6500 $\mathrm{K}$ (altogether 24 different SPDs) were used in the experiment. Altogether 60 observers with normal visual acuity and colour vision participated in the experiment evaluating seven objects related to office environment: a coloured picture, a sample of wood, a smartphone, observers own hand, printed text, a Coke can and a Macbeth Colour checker (MCC) chart (for more details see (Dangol et al., 2013; Islam et al., 2013)).

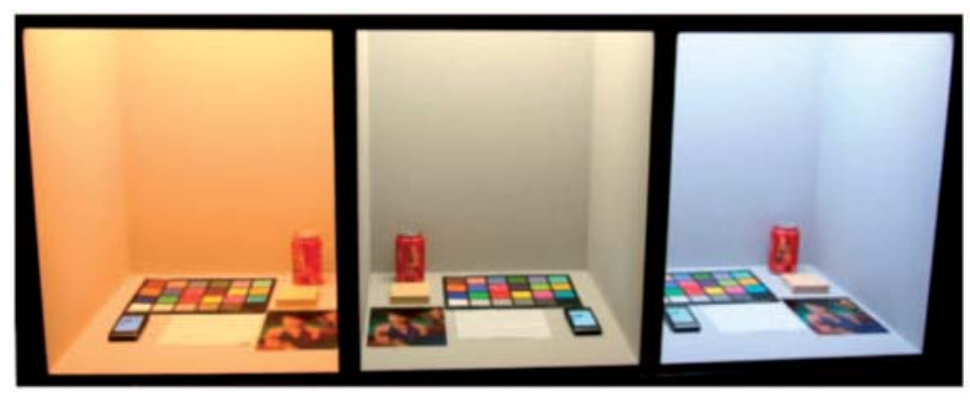

Fig. 2. The three section lighting booth

The questionnaires used in the experiment had two parts: (1) individual evaluation and (2) comparison evaluation. The individual evaluation referred to viewing a single booth at a time and rating the lit environment in that booth by putting a mark on a continuous line scale in the questionnaires. The individual evaluation questions were related to naturalness of the selected seven objects, visual appearance and colourfulness of MCC chart. The comparison evaluation was performed by evaluating the lit scenes under LEDs and FLs where observers compare and rate the naturalness of objects and the overall preferences of the lit scenes. This article deals only with the individual evaluation.

\section{Results}

The 60 observers, 30 male and 30 female aged 20-65 years, evaluated 24 lighting conditions for different questions. The colour characteristic of the 24 lighting sources used in the study are presented in Table 1 . The marked ratings for every question were measured and converted into a range of values between -3 and +3 for statistical analysis. An ANOVA with the significance level of $\mathrm{p}=0.05$ was performed to investigate the statistical significance for the observers' mean rating for particular questions for different SPDs.

The results showed the observers mean ratings are higher for LED SPD2 and LED SPD5 for most of the questions related to the naturalness of objects, the visual conditions of the lit environment and the colourfulness of MCC chart and lower for LED SPD4 and LED SPD6. The summary of the results for different SPDs at $4000 \mathrm{~K}$ is shown in Figure 3 . The detail of statistical analysis are presented in Islam et al.(Islam et al., 2013). The MCRI values for all LED SPDs range from 77 to 95. At CCT of $2700 \mathrm{~K}$, MCRI values for LED SPD1 and LED SPD2 are 89 and 92 respectively. These MCRI values are very close, still observer preferred LED SPD2 to LED SPD1. Similarly, CQS and PS values for LED SPD1 at all CCTs were higher than LED SPD2 at all CCTs, however LED SPD2 were preferred by the observers. PS and CQS values for LED SPD4 and LED SPD6 at all CCTs were mostly lower than other LED SPDs and LED SPD4 and LED SPD6 at all CCTs were least preferred. Moreover, LED SPD2 and LED SPD5 have high FCI values at all CCTs and LED SPD4 and LED SPD6 have lower FCI values. However, LED SPD3 at $6500 \mathrm{~K}$ have higher FCI value than LED SPD2 or LED SPD5 but observer did not prefer LED SPD3. Duv values could be the possible reason why LED SPD3s were not preferred. LED SPD2 or LED SPD5s have negative Duv values and the LED SPD3s have positive Duv values. FCI is area based metric and the above result showed the FCI (area based metric) can used as subjective preference indicator. These results support the idea of Rea and Freyssiner (Rea \& Freyssinier-Nova, 2008) and Guo and Houser(Guo \& Houser, 2004). Similarly, RGAI values for the LED SPD2 and LED SPD5 were higher at all CCTs than other LED SPDs, 
exception being LED SPD5 at $4000 \mathrm{~K}$. The detail comparison between different metrics considered and the mean scores for naturalness of object, visual appearance and colourfulness of MCC chart are shown in Appendix 1.

Table 1. Colour characteristic of the light sources used in the study

\begin{tabular}{|c|c|c|c|c|c|c|c|c|c|c|c|c|}
\hline CCT & & CCT [K] & Duv & CIE CRI & CQS Qp & CQS Qg & PS & MCRI & CQS 9 & FCI & Rg & RGAI \\
\hline \multirow{8}{*}{$2700 \mathrm{~K}$} & LED SPD1 & 2830 & 0,00161 & 98 & 95 & 99 & 97 & 89 & 96 & 129 & 99 & 90 \\
\hline & LED SPD2 & 2803 & $-0,00508$ & 82 & 100 & 119 & 85 & 92 & 87 & 152 & 116 & 109 \\
\hline & LED SPD3 & 2799 & 0.00232 & 82 & 86 & 103 & 89 & 89 & 83 & 135 & 106 & 78 \\
\hline & LED SPD4 & 2766 & 0.00480 & 79 & 71 & 79 & 79 & 79 & 75 & 93 & 88 & 88 \\
\hline & LED SPD5 & 2781 & -0.00286 & 81 & 100 & 117 & 84 & 92 & 89 & 153 & 114 & 111 \\
\hline & LED SPD6 & 2753 & 0.00351 & 81 & 75 & 90 & 94 & 83 & 73 & 119 & 94 & 100 \\
\hline & LED SPD7 & 2744 & -0.00394 & 81 & 81 & 93 & 97 & 83 & 78 & 109 & 95 & 89 \\
\hline & FL & 2663 & 0.00130 & 81 & 79 & 97 & 78 & 77 & 80 & 110 & 101 & 102 \\
\hline \multirow{8}{*}{$4000 \mathrm{~K}$} & LED SPD1 & 4112 & 0,00589 & 97 & 96 & 100 & 92 & 90 & 96 & 119 & 100 & 69 \\
\hline & LED SPD2 & 4097 & $-0,00187$ & 81 & 102 & 116 & 97 & 94 & 89 & 144 & 112 & 72 \\
\hline & LED SPD3 & 4083 & 0,00165 & 82 & 95 & 108 & 98 & 93 & 87 & 135 & 102 & 75 \\
\hline & LED SPD4 & 4116 & 0,00412 & 80 & 79 & 88 & 61 & 84 & 80 & 90 & 92 & 90 \\
\hline & LED SPD5 & 4153 & $-0,00500$ & 80 & 101 & 114 & 98 & 95 & 91 & 144 & 108 & 108 \\
\hline & LED SPD6 & 4120 & 0,00291 & 79 & 76 & 86 & 55 & 83 & 78 & 84 & 92 & 88 \\
\hline & LED SPD7 & 4064 & 0,00343 & 82 & 81 & 92 & 96 & 85 & 79 & 105 & 90 & 97 \\
\hline & FL & 4187 & $-0,00020$ & 79 & 81 & 98 & 81 & 81 & 79 & 104 & 99 & 96 \\
\hline \multirow{8}{*}{$6500 \mathrm{~K}$} & LED SPD1 & 6285 & 0,00434 & 96 & 95 & 101 & 80 & 91 & 94 & 106 & 99 & 85 \\
\hline & LED SPD2 & 6373 & $-0,00096$ & 83 & 102 & 115 & 99 & 95 & 91 & 132 & 110 & 105 \\
\hline & LED SPD3 & 6340 & 0,00562 & 80 & 96 & 113 & 98 & 94 & 84 & 136 & 104 & 73 \\
\hline & LED SPD4 & 6372 & 0,00443 & 83 & 83 & 92 & 68 & 86 & 83 & 89 & 93 & 79 \\
\hline & LED SPD5 & 6355 & 0,00062 & 83 & 101 & 113 & 98 & 94 & 91 & 127 & 110 & 107 \\
\hline & LED SPD6 & 6329 & 0,00458 & 78 & 72 & 84 & 46 & 84 & 75 & 77 & 87 & 72 \\
\hline & LED SPD7 & 6280 & 0,00577 & 81 & 81 & 95 & 67 & 84 & 79 & 91 & 97 & 96 \\
\hline & FL & 6492 & 0,00440 & 79 & 82 & 96 & 68 & 81 & 79 & 92 & 97 & 92 \\
\hline
\end{tabular}

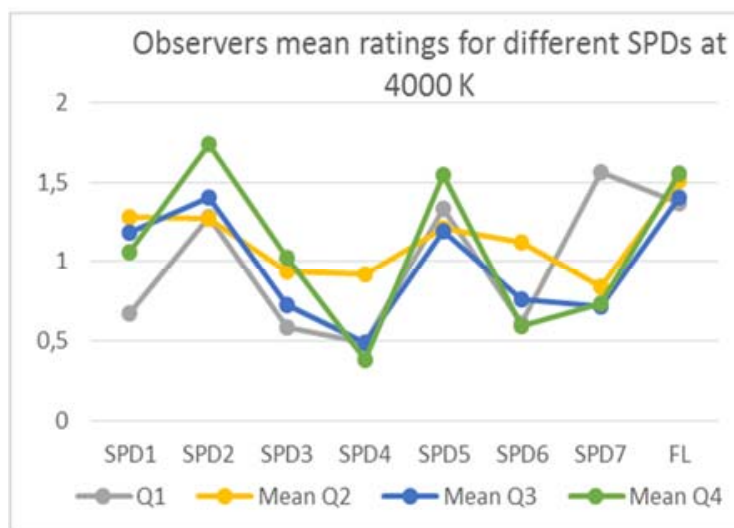

a)

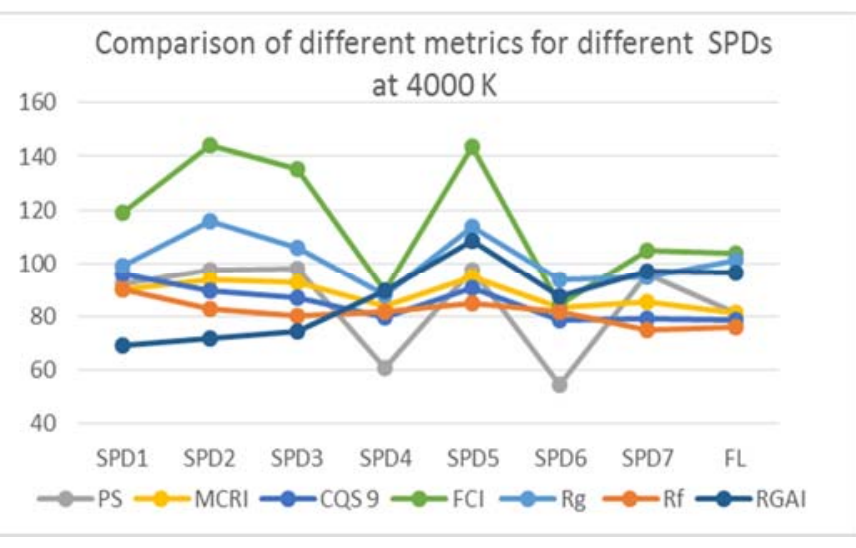

b)

Fig. 3. Comparison of a) observers mean ratings for different SPDs of Q1: naturalness of all objects, Mean Q2: naturalness of objects (average score of mean ratings of six objects), Mean Q3: visual appearance (average score of bright/dim, comfortable/uncomfortable, pleasant/unpleasant, interesting/boring), Mean Q4: Colourfulness of MCC chart (average score of two questions (Dark/bright, discoloured/colourful) and b) different metrics values for different SPDs at $4000 \mathrm{~K}$.

\section{Correlations between mean ratings and different metrics}

The correlation between the mean of observers mean ratings for the naturalness of objects (Q1 and Q2), visual appearance of lit environment (Q3) and colourfulness of MCC chart (Q4) with the PS, MCRI, CQS FCI, Rg and RGAI were analysed with the help of spearman's correlation coefficient (Table 2). The results are presented in Table 2. The results showed that the observers mean ratings on the naturalness of objects (Q1 and Q2) was moderately correlated with Rg, but it was not correlated with the PS, MCRI, CQS, RGAI and FCI.

The observers mean ratings for visual appearance of lit environment were: highly correlated with Rg and moderately correlated with CQS and FCI, but not correlated with PS, MCRI and RGAI. Similarly, the observers mean ratings for colourfulness of MCC chart (Q4) were highly correlated with PS, FCI and Rg, and moderately correlated with CQS and MCRI. 
Table 2 Spearman's correlation coefficient between the metrics (PS, MCRI, CQS, FCI, Rg, RGAI) and observers mean ratings for different questions

\begin{tabular}{|c|c|c|c|c|c|c|c|c|c|}
\hline \multicolumn{10}{|c|}{ Correlations between average mean ratings of different questions and metrics } \\
\hline & $\begin{array}{l}\text { Mean of } \\
\text { Q1 \& Q2 }\end{array}$ & $\begin{array}{c}\text { Mean of } \\
\text { Q3 }\end{array}$ & $\begin{array}{c}\text { Mean of } \\
\text { Q4 }\end{array}$ & PS & MCRI & CQS9 & FCI & Rg & RGAI \\
\hline $\begin{array}{l}\text { Mean of } \\
\text { Q1 \& Q2 }\end{array}$ & 1 & $0,894^{* *}$ & $0,799^{* *}$ & 0,17 & 0,078 & 0,302 & 0,228 & $0,480^{*}$ & 0,245 \\
\hline $\begin{array}{c}\text { Mean of } \\
\text { Q3 } \\
\end{array}$ & & & $0,923^{* *}$ & 0,374 & 0,366 & $0,425^{*}$ & $0,408^{*}$ & $0,632^{* *}$ & 0,182 \\
\hline $\begin{array}{c}\text { Mean of } \\
\text { Q4 }\end{array}$ & & & & $0,524^{* *}$ & $0,493^{*}$ & $0,487^{*}$ & $0,595^{* *}$ & $0,793^{* *}$ & 0,346 \\
\hline PS & & & & & $0,674^{* *}$ & $0,521^{* *}$ & $0,750^{* *}$ & $0,591^{* *}$ & 0.142 \\
\hline MCRI & & & & & & $0,779^{* *}$ & $0,698^{* *}$ & $0,703^{* *}$ & 0,039 \\
\hline CQS9 & & & & & & & $0,604^{* *}$ & $0,689^{* *}$ & 0,04 \\
\hline FCI & & & & & & & & $0,883^{* *}$ & 0,256 \\
\hline Rg & & & & & & & & & 0,316 \\
\hline $\begin{array}{l}\text { Correlatic } \\
\text { * Correlati }\end{array}$ & $\begin{array}{l}\text { is signifi } \\
\text { is signif }\end{array}$ & $\begin{array}{l}\text { icant at tl } \\
\text { ficant at }\end{array}$ & $\begin{array}{l}.05 \text { level } \\
0.01 \text { level }\end{array}$ & $\begin{array}{l}\text { tailed) } \\
\text {-tailed) }\end{array}$ & & & & & \\
\hline
\end{tabular}

\section{Conclusions}

The results showed that among the metrics considered, Rg, RGAI and FCI were able to predict the best LED SPDs which can better define the subject assessment for the naturalness of object, visual appearances and colourfulness (Fig. A-1 - Fig. A-6). The Rg, RGAI and FCI values for LED SPD2 and LED SPD5 at all CCTs were higher than other LED SPDs and these LED SPDs (LED SPD2 and LED SPD5) were most preferred by the observers. LED SPD4 and LED SPD6 at most CCTs (considered) have relatively low FCI, RGAI and Rg values and were least preferred. It is worth to mention that all LED SPDs except LED SPD1 at all CCTs have CIE CRI values of around 80. The Spearman's correlation coefficient showed that the Rg was moderately correlated with the observers mean ratings for naturalness of object, and Rg was highly correlated with the observers mean ratings for visual appearance and the colourfulness of MCC chat. Whereas, FCI and CQS were moderately correlated with the observers mean ratings for visual appearance and all other metrics considered, except RGAI, were moderately correlated with the observers mean rating for colourfulness of MCC chart. Therefore, this study showed that the gamut area based metrics like Rg, and FCI are applicable to predict the subjective preference (in terms of naturalness, colourfulness and visual appearance) rank order of a set of LED SPDs when all LED SPDs in that set have close CIE CRI values. In addition, it was found that chromaticity difference (Duv) values of the spectra affected the observers' preference. The observers preferred the LED SPDs whose Duv values were either negative or close to the black body locus.

\section{References}

CIE. (1995). Method of Measuring and Specifying Colour Rendering Properties of Light Sources. Vienna, Austria, CIE Technical Report, CIE 13.3: 1995.

Cree. (2014). Cree Shatters Efficiency Benchmark with First 200-Lumen-Per-Watt LED Luminaire. Retrieved March 2, 2017, from //www.cree.com/News-and-Events/Cree-News/PressReleases/2014/January/200-LPW-fixture

Dangol, R., Islam, M., Hyvärinen, M., Bhusal, P., Puolakka, M., Halonen, L., ... Halonen, L. (2013). Subjective preferences and colour quality metrics of LED light sources. Lighting Research and Technology, 45(6), 666-688. https://doi.org/10.1177/1477153512471520

David, A., Fini, P. T., Houser, K. W., Ohno, Y., Royer, M. P., Smet, K. A. G., ... Whitehead, L. (2015). Development of the IES method for evaluating the color rendition of light sources. Optics Express, 23(12), 15888. https://doi.org/10.1364/OE.23.015888

Davis, W., \& Ohno, Y. (2010). Color quality scale. Optical Engineering, 49(3), 1-16. https://doi.org/10.1117/1.3360335

Guo, X., \& Houser, K. W. W. (2004). A review of colour rendering indices and their application to commercial light sources. Lighting Research and Technology, 36(3), 183-199. https://doi.org/10.1191/1365782804li112oa

Hashimoto, K., \& Nayatani, Y. (1994). Visual clarity and feeling of contrast. Color Research \& Application, 
19(3), 171-185. https://doi.org/10.1002/col.5080190305

Hashimoto, K., Yano, T., Shimizu, M., \& Nayatani, Y. (2007). New method for specifying colour rendering properties of light sources based on feeling of contrast. Color Research and Application, 32(5), 361371.

Illuminating Engineering Society (IES). (2015). IES Method for Evaluating Light Source Color Rendition: IES TM-30-15 (IES TM-30-). New York: The Illuminating Engineering Society of North America. Retrieved from https://books.google.nl/books?id=2z0CjwEACAAJ

Islam, M., Dangol, R., Hyvärinen, M., Bhusal, P., Puolakka, M., \& Halonen, L. (2013). User preferences for LED lighting in terms of light spectrum. Lighting Research \& Technology, 45(6), 641-665. https://doi.org/10.1177/1477153513475913

Li, C., Luo, M. R., Rigg, B., \& Hunt, R. W. G. (2002). CMC 2000 chromatic adaptation transform: CMCCAT2000. Color Research \& Application, 27(1), 49-58. https://doi.org/10.1002/col.10005

Rea, M. S., \& Freyssinier-Nova, J. P. (2008). Color rendering: A tale of two metrics. Color Research \& Application, 33(3), 192-202. https://doi.org/10.1002/col.20399

Smet, K., Ryckaert, W., Pointer, M., Deconinck, G., \& Hanselaer, P. (2012). Optimization of colour quality of LED lighting with reference to memory colours. Lighting Research and Technology, 44(1), 7-15. https://doi.org/10.1177/1477153511432250

Smet, K., Ryckaert, W. R., Pointer, M. R., Deconinck, G., \& Hanselaer, P. (2010). Memory colours and colour quality evaluation of conventional and solid-state lamps. Optics Express, 18(25), 26229-26244. https://doi.org/10.1002/col.20620

Smet, K., Ryckaerta, W., Deconinckb, G., \& Hanselaera, P. (2010). A colour rendering metric based on memory colours (MCRI). Optics Express, 18(25), 438-440. Retrieved from http://www.create.uwe.ac.uk/norway_paperlist/smet.pdf

Teunissen, C., Van Der Heijden, F., Phd, P., \& De Beer, E. (n.d.). Characterising user preference for white LED light sources with CIE colour rendering index combined with a relative gamut area index. https://doi.org/10.1177/1477153515624484

Yano, T., \& Hashimoto, K. (2015). Preference index for Japanese complexion under illuminations. Color Research \& Application, n/a-n/a. https://doi.org/10.1002/col.21948 


\section{Appendix 1}

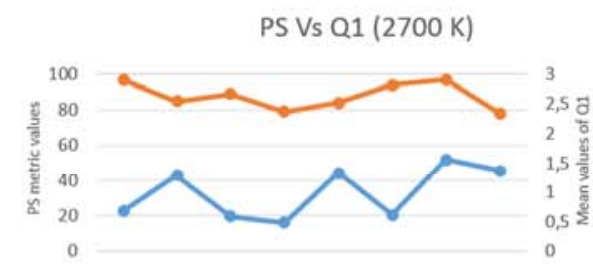

SPD1 SPD2 SP03 SPD4 SPDS SPD6 SPD7 F1

$$
\rightarrow \text { PS } \rightarrow \text { Q1 }
$$

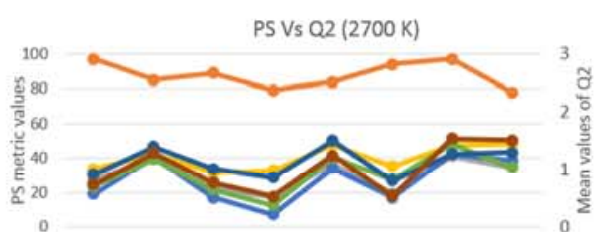

SPD1 SPD2 SPD3 SPD4 SPD5 SPD6 SPD7 $\rightarrow-P S \rightarrow 02.1 \rightarrow 02.2 \rightarrow 02.3$

$\rightarrow 024 \rightarrow 025 \rightarrow 026$

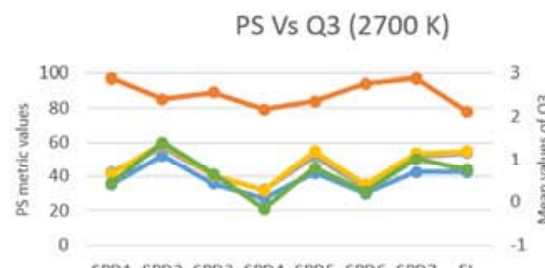

SPO1 SPD2 SPD3 SPO4 SPO5 SPD6 SPO7 FL

$\rightarrow \mathrm{PS} \rightarrow \mathrm{Q} 3.4 \rightarrow \mathrm{Q} 3.3 \rightarrow \mathrm{Q} 3.2 \rightarrow \mathrm{Q} 3.1$

)

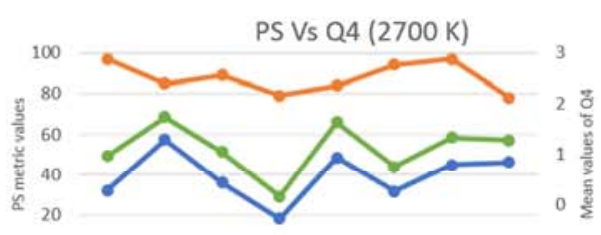

SPD1 $\quad$ SPD2 $\quad$ SPD3 SPD4 SPD5 SPD6 SPD7

$\rightarrow$ PS $\rightarrow 04.1 \rightarrow 04.2$

(a)

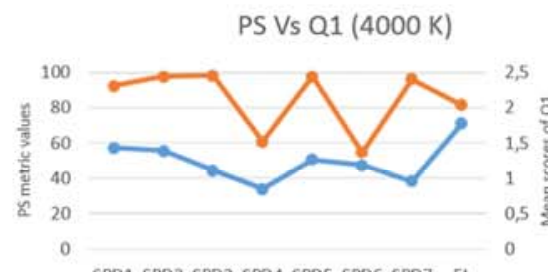

SPD1 SPD2 SPD3 SPD4 SPDS SPD6 SPD7 FL $\rightarrow P S \rightarrow Q 1$

PS Vs Q1 (6500 K)

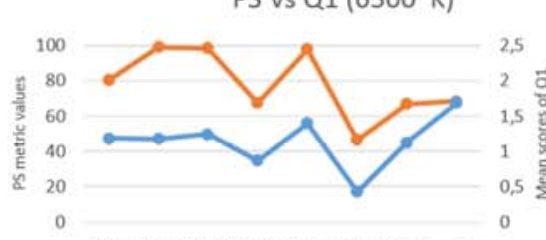

SPD1 SPD2 SPD3 SPD4 SPDS SPD6 SPD7 FI

$$
\rightarrow P S \rightarrow Q 1
$$

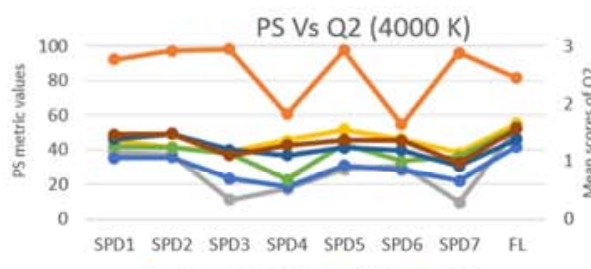

$\rightarrow \mathrm{Ps} \rightarrow \mathrm{O} 2.1 \rightarrow \mathrm{Q} 2.2 \rightarrow \mathrm{Q} 2.3$

$\rightarrow 02.4 \rightarrow 02.5 \rightarrow 02.6$

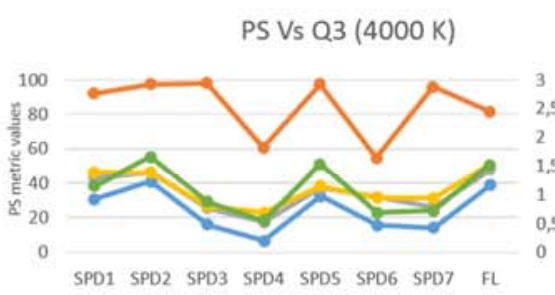

SPD1 SPD2 SPD3 SPD4 SPDS SPD6 SPD7 FL

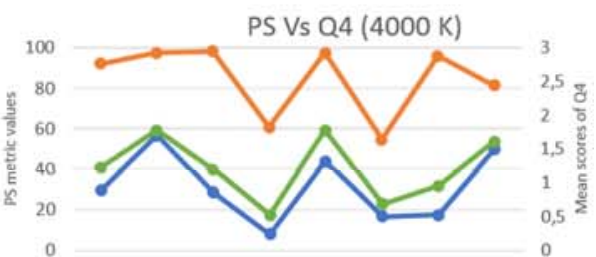

SPD1 1 SPD2 SPD3 SPD4 SPDS SPD6 SPD7 FL

$$
\rightarrow P S \rightarrow Q 4.1 \rightarrow Q 4.2
$$

(b)

\section{- PS Vs $03(6500 \mathrm{~K})$}

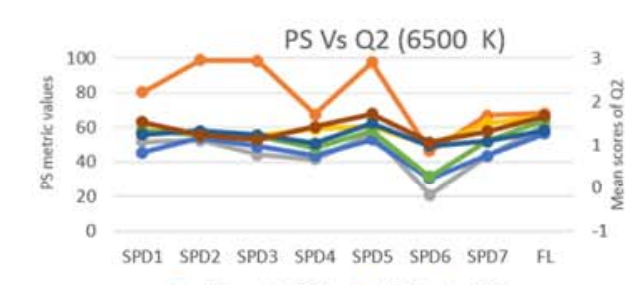

$\rightarrow \mathrm{PS} \rightarrow \mathrm{Q} 2.1 \rightarrow \mathrm{Q} 2.2 \rightarrow \mathrm{Q} 2.3$

$\rightarrow 02.4 \rightarrow 02.5 \rightarrow 02.6$
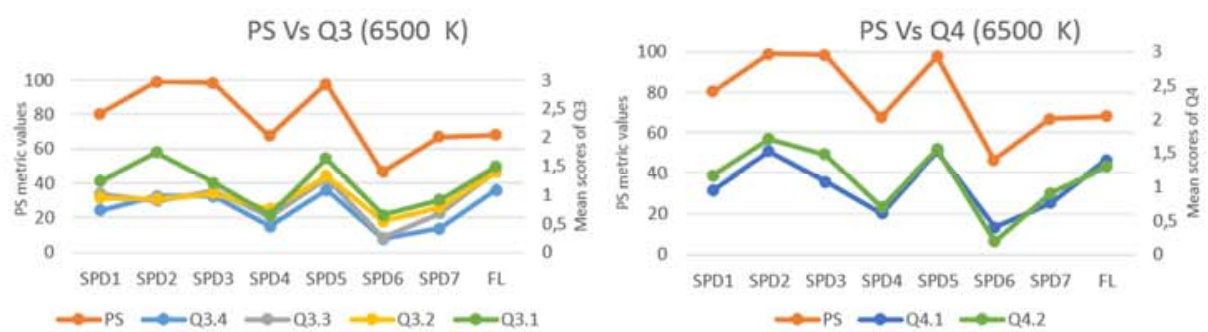

$\rightarrow$ PS $\rightarrow$ Q O4.1 $\rightarrow$ Q 4.2

(c)

Fig. A-1. Comparison of preference of skin (PS) metric values (left vertical axis) with the mean scores (right vertical axis) of Q1: naturalness of all objects, Q2: naturalness of objects (score of mean ratings of six objects), Q3: visual appearance (score of bright/dim, comfortable/uncomfortable, pleasant/unpleasant, interesting/boring), Q4: Colourfulness of MCC chart (score of two questions (Dark/bright, discoloured/colourful) under different SPDs at (a) $2700 \mathrm{~K}$, (b) $4000 \mathrm{~K}$, and (c) $6500 \mathrm{~K}$ 


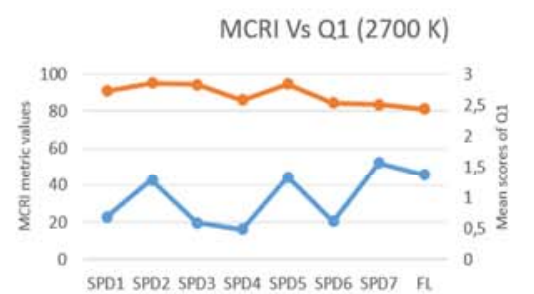

$\rightarrow \mathrm{MCHI} \rightarrow \mathrm{Q} 1$

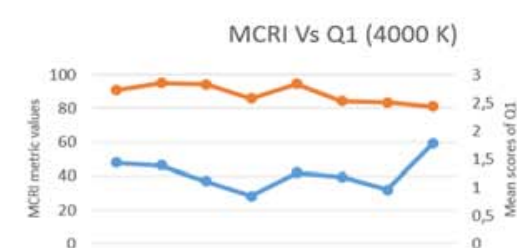

SPD1 SPO2 SPO3 SPD4 SPDS SPD6 SPD7 F

$\rightarrow$ MCR1 $\rightarrow$ Q 1

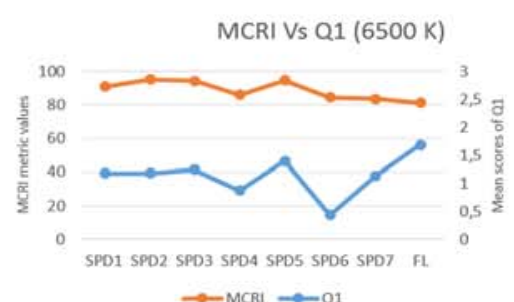

$\rightarrow-M C R 1 \rightarrow-\alpha$

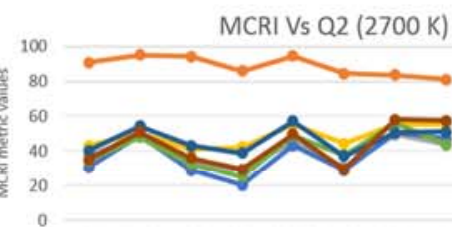

SP01 SPD2 SPD3 SPD4 SPD5 SPD6 SPD7

$\rightarrow$ MCRI $-002.12=0.22-002.3$

$\rightarrow 02.4 \rightarrow 02.5 \rightarrow 02.6$

MCRI Vs Q2 (4000 K)

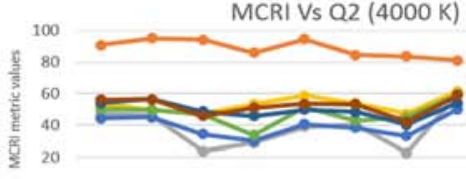

SPD1 SPO2 SP03 SPD4 SPOS SPO6 SPD7

$\rightarrow$ MCRI $\rightarrow 02.1 \rightarrow 02.2 \rightarrow 02.3$

$\rightarrow 02.4 \rightarrow 02.5 \rightarrow 02.6$

MCRI Vs Q2 (6500 K)

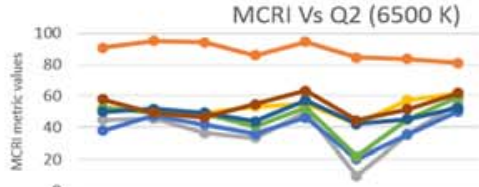

SPD1 SPD2 SPD3 SPP4 SPD5 SPD6 SP07

$\rightarrow$ MCRI $\rightarrow 02.1 \rightarrow 02.2 \div 02$

$\rightarrow 02.4 \rightarrow 02.5 \rightarrow 02.6$

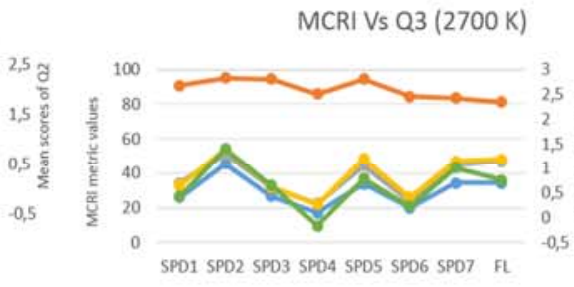

$\rightarrow$ MCP1 $\rightarrow 03.4 \rightarrow 03.3 \rightarrow 03.2 \rightarrow 03$

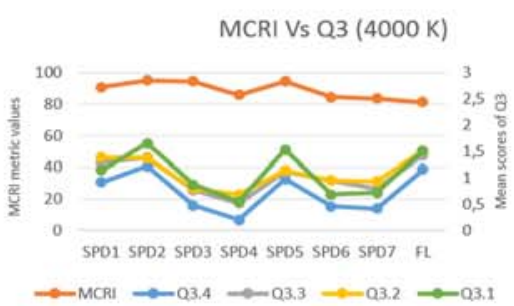

(b)

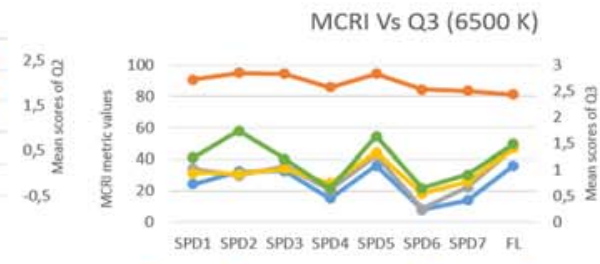

(c)

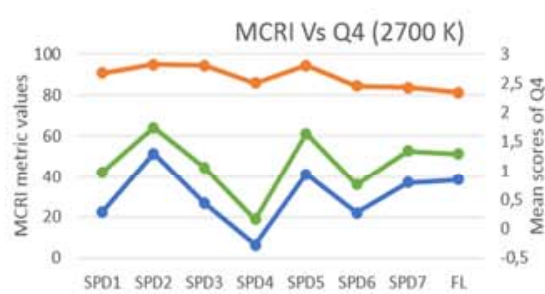

$\rightarrow$ MCRI $\rightarrow 04.1 \rightarrow 04.7$

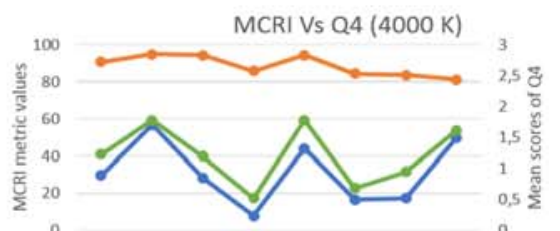

SPO1 SPD2 SPD3 SPD4 SPD5 SPD6 SPD7 FL

$\rightarrow$ MCR1 $\rightarrow 04.1 \rightarrow 04.2$

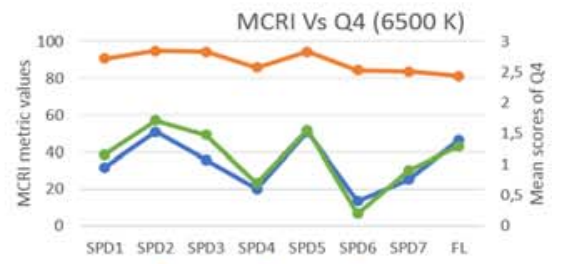

$\rightarrow$ MCR1 $\rightarrow$ Q4.1 $\rightarrow$ Q Q4.2

Fig. A-2. Comparison of Memory colour rendering index (MCRI) values (left vertical axis) with the mean scores (right vertical axis) of Q1: naturalness of all objects, Q2: naturalness of objects (score of mean ratings of six objects), Q3: visual appearance (score of bright/dim, comfortable/uncomfortable, pleasant/unpleasant, interesting/boring), Q4: Colourfulness of MCC chart (score of two questions (Dark/bright,

discoloured/colourful) under different SPDs at (a) $2700 \mathrm{~K}$, (b) $4000 \mathrm{~K}$, and (c) $6500 \mathrm{~K}$ 


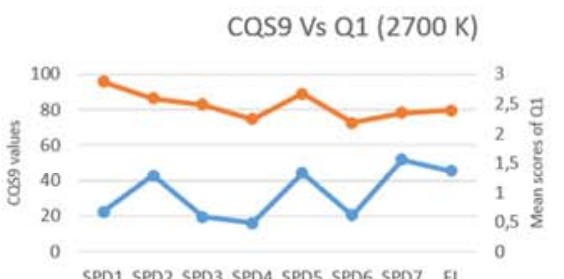

SPD1 SPD2 SPD3 SPD4 SPD5 SPD6 SPD7 FL $\rightarrow \cos 9 \rightarrow 01$

cQ59 Vs Q1 (4000 K)

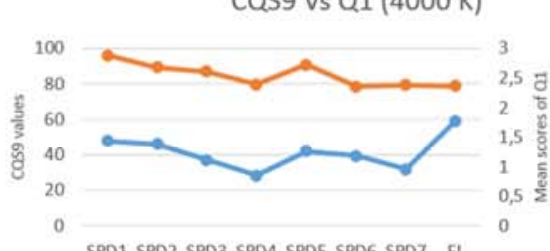

SPD1 SPD2 SPD3 SPD4 SPD5 SPD6 SPD7 FI $\rightarrow \cos 9 \rightarrow 01$

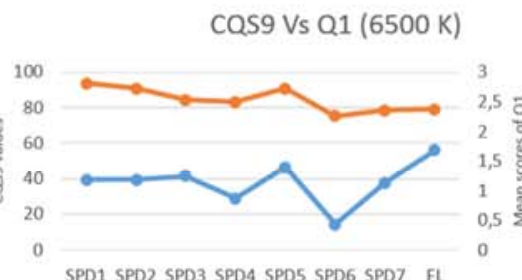

SPD1 SPD2 SP03 SPD4 SPD5 SPD6 SPD7 FL $\rightarrow \cos 9 \rightarrow 01$

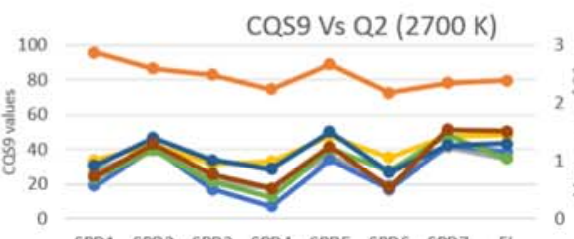

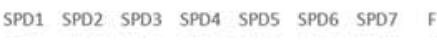
$\rightarrow \cos 9 \rightarrow 02.1 \rightarrow 02.2 \rightarrow 02.3$

$\rightarrow 02.4 \rightarrow 02.5 \rightarrow 02.6$

cQs9 Vs Q2 (4000 K)

$$
\begin{array}{r}
100 \\
80 \\
\frac{0}{3} 60 \\
58 \\
80 \\
20 \\
0
\end{array}
$$

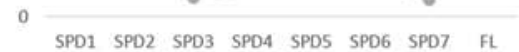

$\rightarrow \cos 9 \rightarrow 02.1 \rightarrow 02.2 \rightarrow 02.3$

$\rightarrow 02.4 \rightarrow 02.5 \rightarrow 02.6$

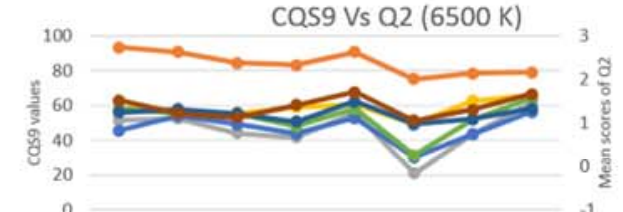

SPD1 $\quad$ SPD2 SPD3 SPD4 SPO5 SPO6 $\quad$ SPD7 $\quad \mathrm{FL}$ $\rightarrow \cos 9 \rightarrow 02.1 \rightarrow 02.2 \rightarrow 02.3$

$\rightarrow 02.4 \rightarrow 02.5 \rightarrow 02.6$

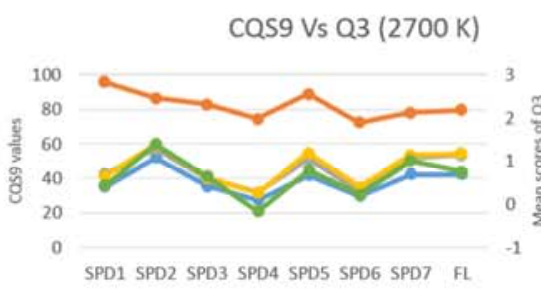

$-\cos 9 \rightarrow 03.4 \rightarrow 03.3 \rightarrow 03.03 .03$.

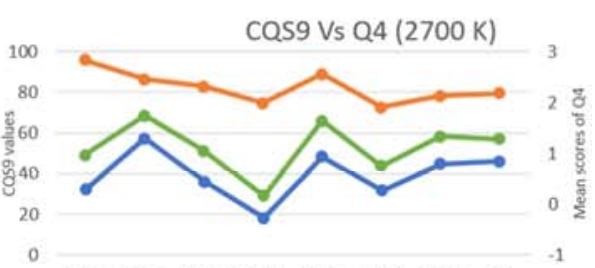

SPD1 SPO2 SP03 SPD4 SPO5 SPD6 SPO7 FL.

$\rightarrow \operatorname{coss} \rightarrow 04.1 \rightarrow 04.2$

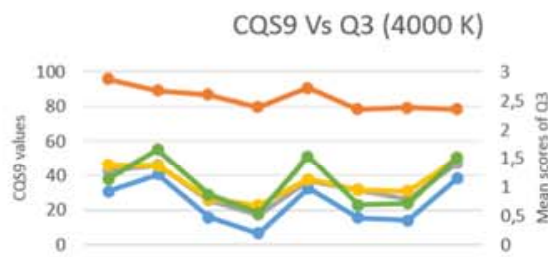

SPD1 SPD2 SPD3 SPD4 SPD5 SPD6 SPD7 FL

$\rightarrow \cos 9 \rightarrow 03.4 \rightarrow 03.3 \rightarrow 03.2 \rightarrow 03.1$

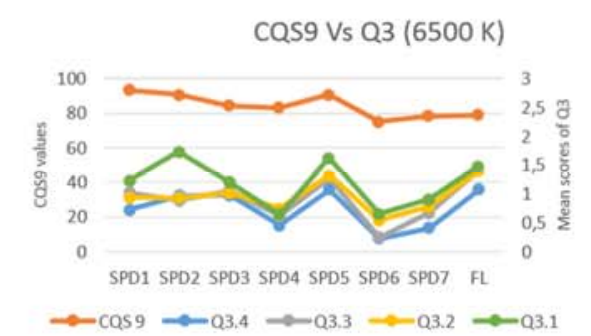

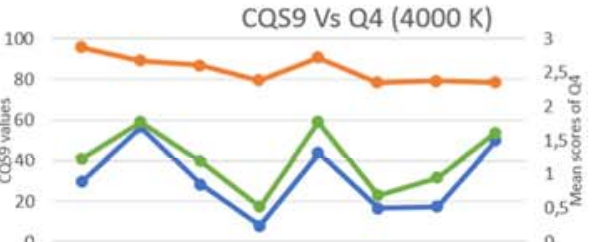

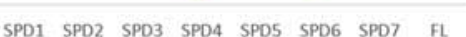

$\rightarrow \cos 9 \rightarrow 04.1 \rightarrow Q 4.2$

(c)

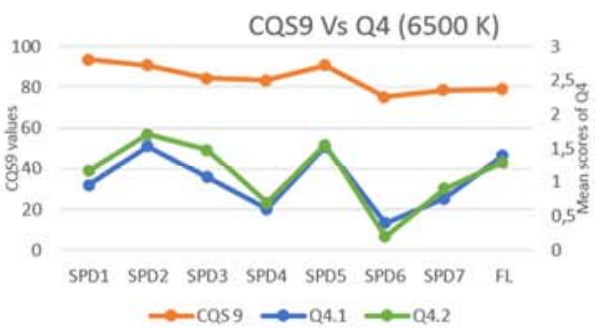

Fig. A-3. Comparison of colour quality scale (CQS version 9) values (left vertical axis) with the mean scores (right vertical axis) of Q1: naturalness of all objects, Q2: naturalness of objects (score of mean ratings of six objects), Q3: visual appearance (score of bright/dim, comfortable/uncomfortable, pleasant/unpleasant, interesting/boring), Q4: Colourfulness of MCC chart (score of two questions (Dark/bright, discoloured/colourful) under different SPDs at (a) $2700 \mathrm{~K}$, (b) $4000 \mathrm{~K}$, and (c) $6500 \mathrm{~K}$ 


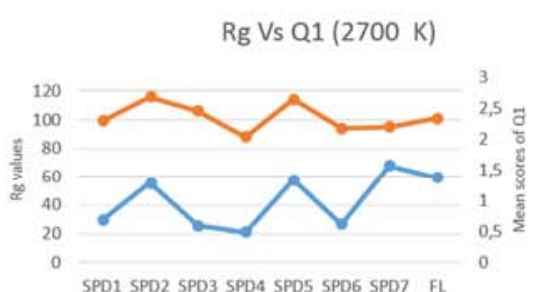

$\rightarrow-R_{8} \rightarrow 01$

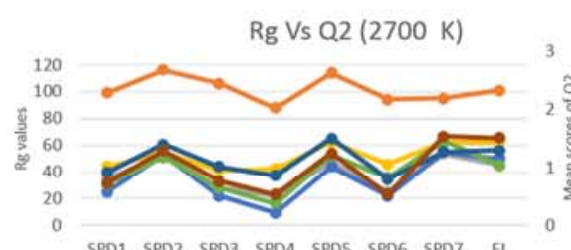

SP01 SPD2 SPD3 SPD4 SPD5 SPO6 SPD7

$\rightarrow \mathrm{Rg} \rightarrow \mathrm{Q} 2.1 \mathrm{Q} \rightarrow \mathrm{Q} 2.2 \rightarrow 02.3$

$\rightarrow 02.4 \rightarrow 02.5 \rightarrow 02.6$

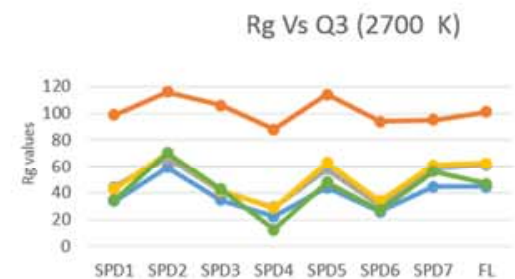

$\rightarrow \mathrm{Rg} \rightarrow \mathrm{O} 3.4 \rightarrow \mathrm{Q} 3.3 \rightarrow \mathrm{Q} 3.2 \rightarrow \mathrm{O} 3.1$
Rg Vs Q4 (2700 K)

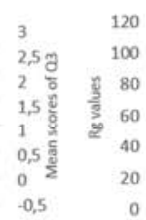

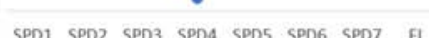

$\rightarrow \mathrm{Rg} \rightarrow \mathrm{Q} 4.1 \rightarrow 04.2$
Rg Vs Q1 (4000 K)

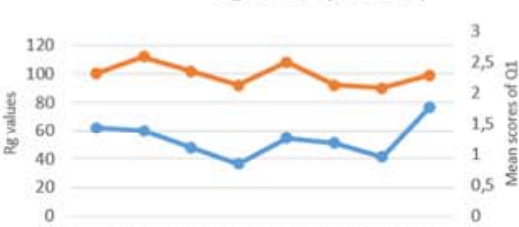

SPD1 SPD2 SPD3 SP04 SPD5 SPD6 SPD7 F1

$\rightarrow \mathrm{RB}_{\mathrm{B}} \rightarrow \mathrm{Q}_{1}$

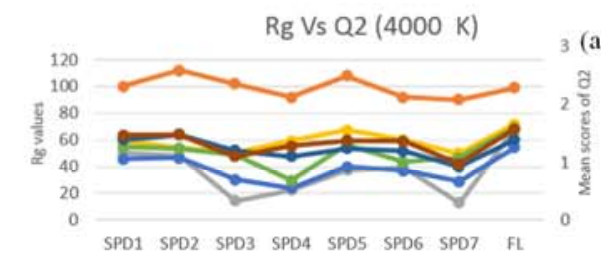

$\rightarrow \mathrm{Rg} \rightarrow \mathrm{Q} 2.1 \rightarrow \mathrm{Q} 2.2 \rightarrow \mathrm{O} 2.3$

$\rightarrow 024 \rightarrow 02.502$
Rg Vs Q3 (4000 K)

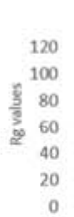

20

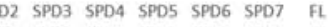

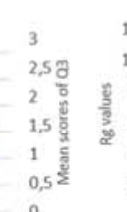

Q3.1
Rg Vs Q4 (4000 K)

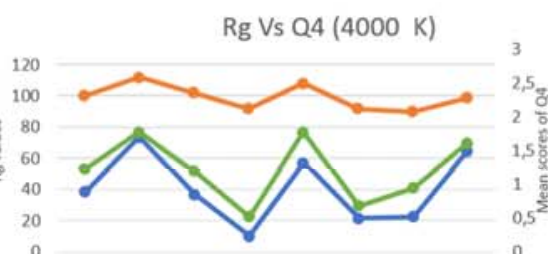

SPD1 5 SPD2 SPD3 SPD4 SPDS SPD6 SPD7

$\rightarrow R_{8} \rightarrow 04.1 \rightarrow 04.2$
Rg Vs Q1 (6500 K)

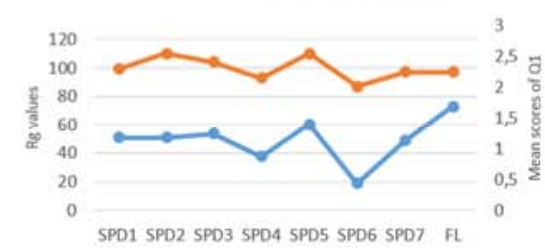

$\rightarrow R_{B} \rightarrow Q_{1}$
Rg Vs Q2 (6500 K)

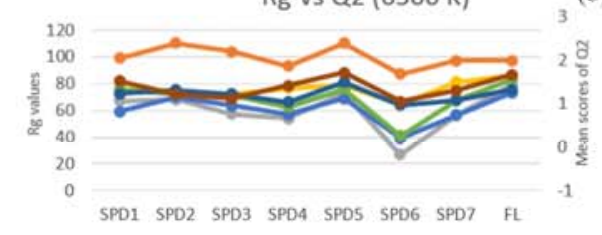

$\rightarrow \mathrm{Rg} \rightarrow \mathrm{Q} 2.1 \rightarrow 02.2 \rightarrow 02$.
Rg Vs Q3 (6500 K)
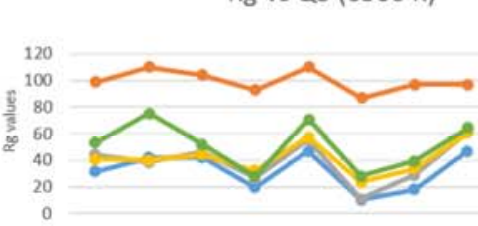

SP01 SPD2 SP03 SP04 SPOS SPO6 SP07 FL

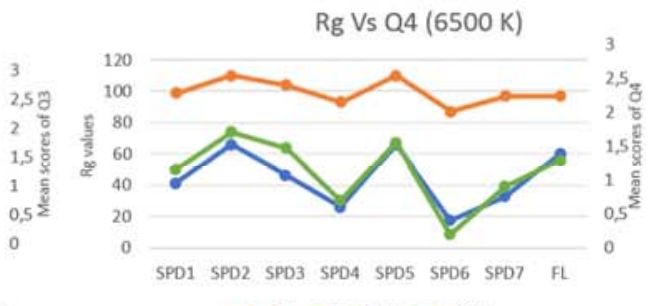

$\rightarrow \mathrm{Rg} \rightarrow \mathrm{Q} 4.1 \rightarrow 04.2$

Fig. A-4. Comparison of Rg values (left vertical axis) with the mean scores (right vertical axis) of Q1: naturalness of all objects, Q2: naturalness of objects (score of mean ratings of six objects), Q3: visual appearance (score of bright/dim, comfortable/uncomfortable, pleasant/unpleasant, interesting/boring), Q4: Colourfulness of MCC chart (score of two questions (Dark/bright, discoloured/colourful) under different SPDs at (a) 2700 $\mathrm{K}$, (b) $4000 \mathrm{~K}$, and (c) $6500 \mathrm{~K}$ 


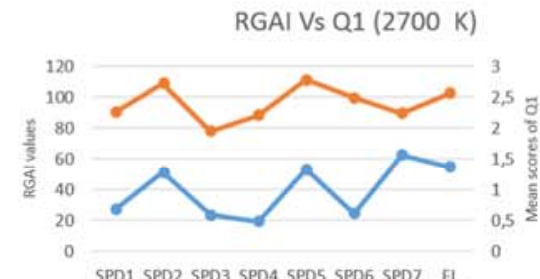

SPD1 SPD2 SPD3 SPD4 SPDS SPD6 SPD7 FL $\rightarrow$ RGAI $\rightarrow 01$

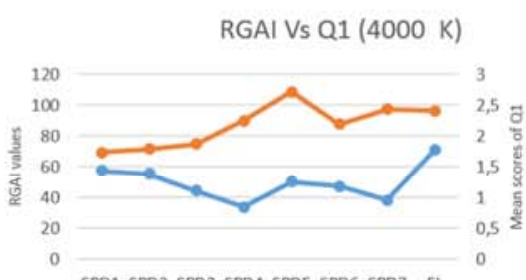

$\rightarrow$ RGAI $\rightarrow 0$

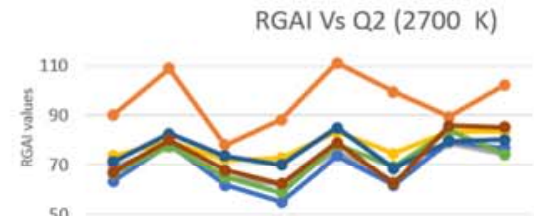

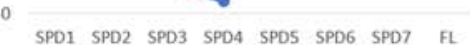
$\rightarrow$ RGAI $\rightarrow 02.1 \rightarrow 02.2 \rightarrow 02.3$ $\rightarrow 02.4 \rightarrow 02.5 \rightarrow 02.6$

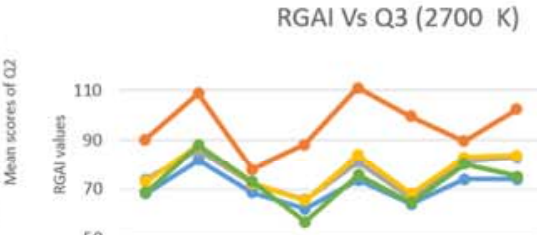

50 SP01 SPO2 SPO3 SPD4 SPOS SPO6 SPO7 FL $\rightarrow$ RGAI $\rightarrow 03.4 \rightarrow 03.3 \rightarrow 03.3 \rightarrow 03.4$
RGAI Vs Q4 (2700 K)

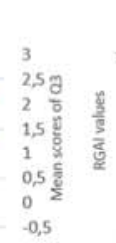

SPD1 SPD2 SPD3 SPO4 SPD5 SPD6 SPD7 FL $\rightarrow$ RGAI $\rightarrow 04.1 \rightarrow 04.2$
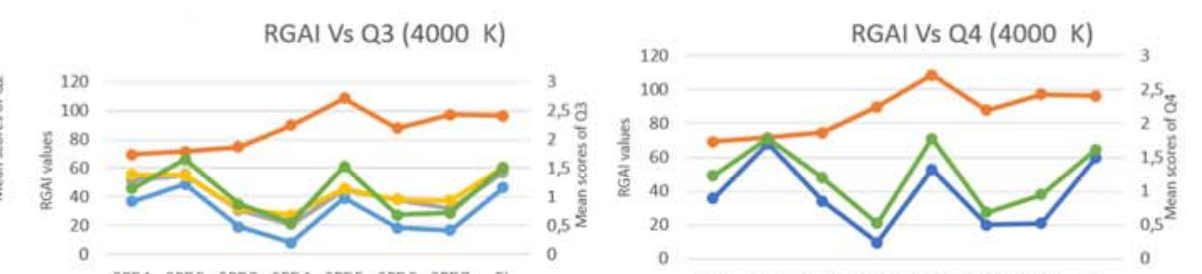

SPD1 SPD2 SPO3 SPD4 SPOS SPD6 SPD7 FL

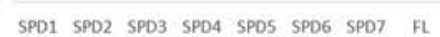
$\rightarrow$ RGAI $\rightarrow$ Q Q 4.1 $\rightarrow$ Q Q 4.2

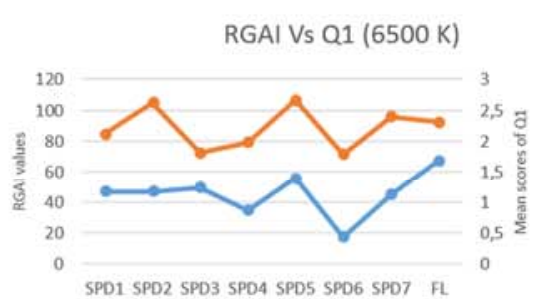

$\rightarrow$ RGAI $\rightarrow$ Q1

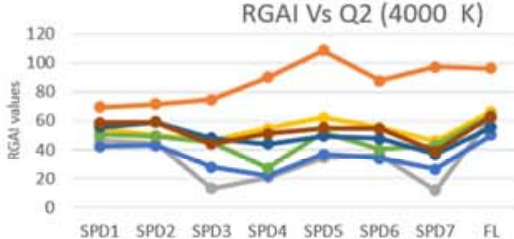

$\Rightarrow \mathrm{RGA1} \rightarrow \mathrm{O22.1} \rightarrow \mathrm{O2.2} \rightarrow 02.2$ $\rightarrow$ RGAI $\rightarrow$ Q3.4 $\rightarrow$ Q3.3 $\rightarrow$ Q3.2 $\rightarrow$ Q3.1

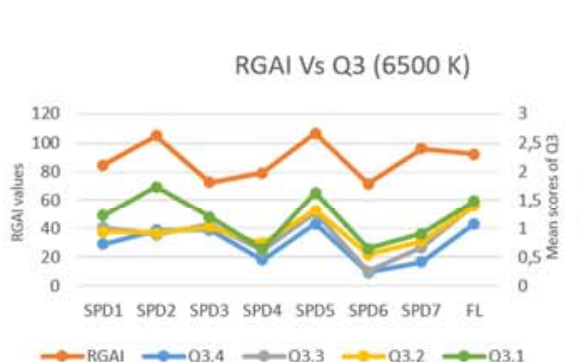

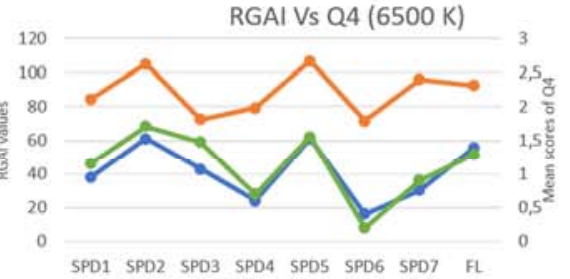

$\rightarrow$ RGAI $\rightarrow 04.1 \rightarrow 04.2$

(c)

Fig. A-5. Comparison Relative gamut area index (RGAI) values (left vertical axis) with the mean scores (right vertical axis) of Q1: naturalness of all objects, Q2: naturalness of objects (score of mean ratings of six objects), Q3: visual appearance (score of bright/dim, comfortable/uncomfortable, pleasant/unpleasant, interesting/boring), Q4: Colourfulness of MCC chart (score of two questions (Dark/bright, discoloured/colourful) under different SPDs at (a) $2700 \mathrm{~K}$, (b) $4000 \mathrm{~K}$, and (c) $6500 \mathrm{~K}$ 


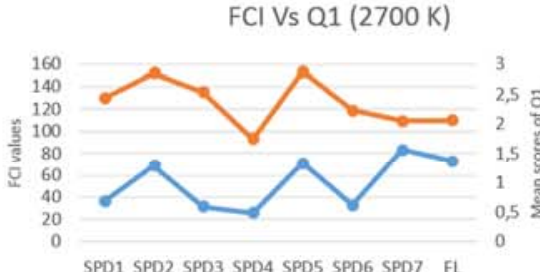

$\rightarrow-\mathrm{FC} \rightarrow \mathrm{Q1}$

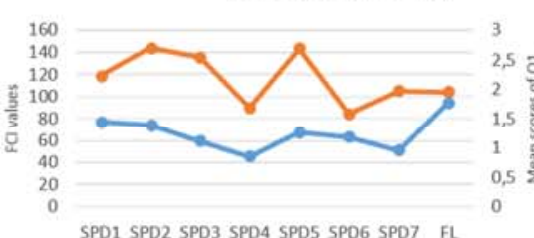

$\rightarrow-\mathrm{FC} \rightarrow \mathrm{Q} 1$

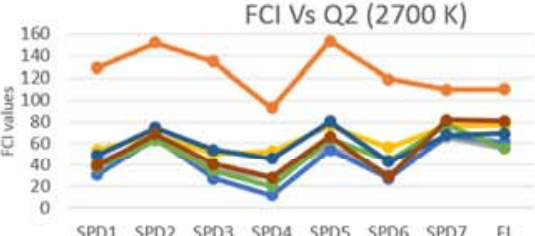

SPO1 SPD2 SPP3 SPD4 SPDS SPDE SPD7 -

$\rightarrow \mathrm{FCl} \rightarrow \mathrm{Q2.1} \rightarrow \mathrm{Q} 2.2 \rightarrow \mathrm{Q} 2.3$

$\rightarrow 02.4 \rightarrow 02.5 \rightarrow 02.6$

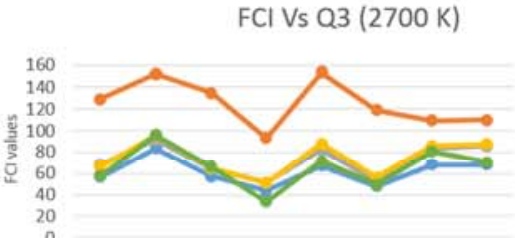

SPD1 $\quad$ SPD2 $\quad$ SPD3 SPD4 SPD5 SPD6 SPD7 FL

$\rightarrow \mathrm{FCl} \rightarrow \mathrm{Q} 3.4 \rightarrow \mathrm{Q} 3.3 \rightarrow \mathrm{Q} 3.2 \rightarrow \mathrm{Q} 3.1$

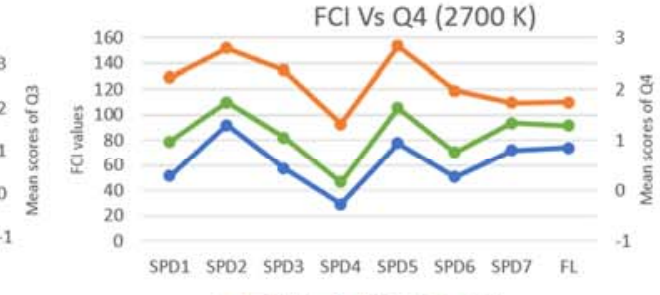

$\rightarrow \mathrm{FCO} \rightarrow \mathrm{Q} 4.1 \rightarrow 04.2$

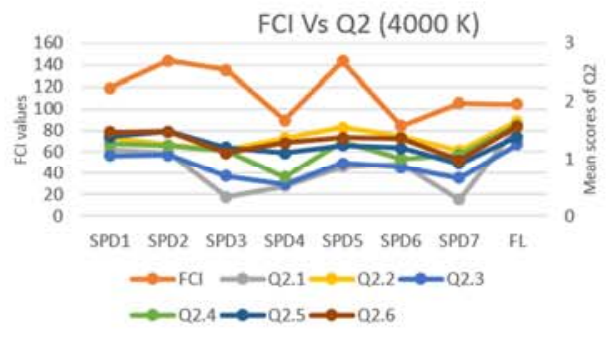

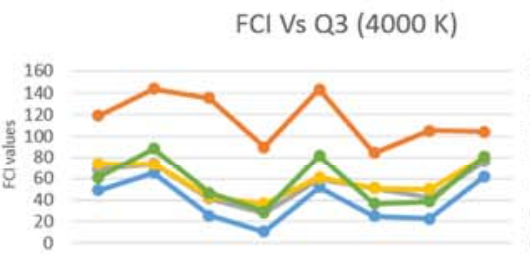

SPD1 $\quad$ SPD2 $\quad$ SPD3 SPD4 SPD5 SPD6 SPD7 FL

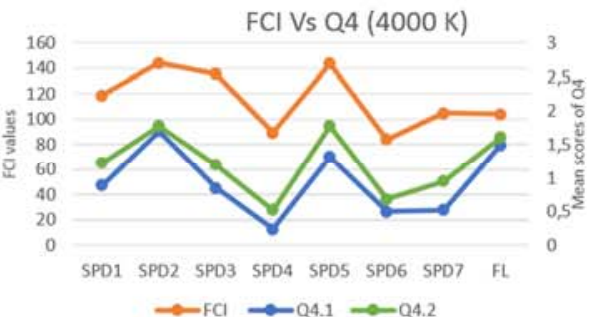

$\rightarrow \mathrm{FCl} \rightarrow \mathrm{Q} 4.1 \rightarrow \mathrm{Q} 4.2$

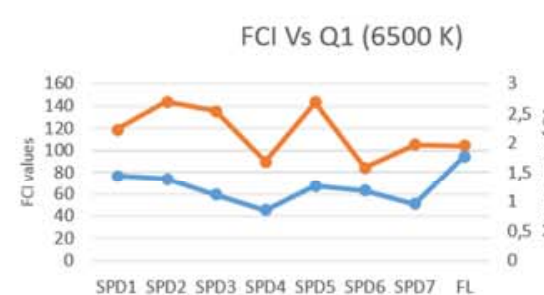

$$
\rightarrow \mathrm{FC} \rightarrow \mathrm{Q} 1
$$

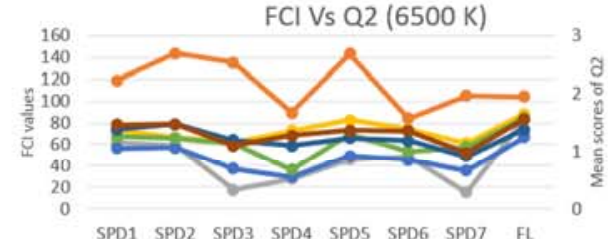

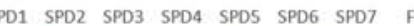
$\rightarrow \mathrm{FCl} \rightarrow 02.1 \rightarrow 02.2 \rightarrow 023$

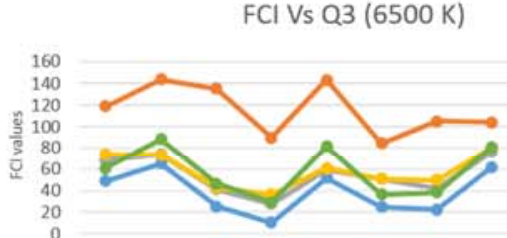

SPD1 SPD2 SPD3 SPD4 SPD5 SPDE SPD7

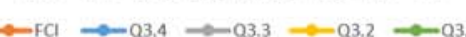

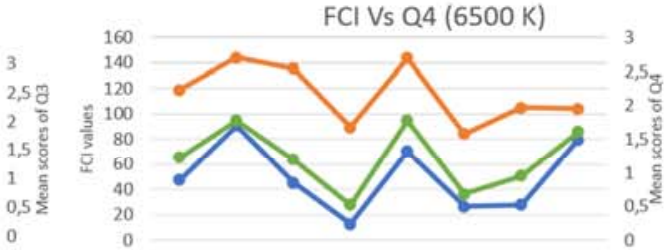

SPD1 SPD2 SPD3 SPD4 SPD5 SPD6 SPD7 FL

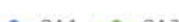

Fig. A-6. Comparison Feeling of contracts index (FCI) values (left vertical axis) with the mean scores (right vertical axis) of Q1: naturalness of all objects, Q2: naturalness of objects (score of mean ratings of six objects), Q3: visual appearance (score of bright/dim, comfortable/uncomfortable, pleasant/unpleasant, interesting/boring), Q4: Colourfulness of MCC chart (score of two questions (Dark/bright, discoloured/colourful) under different SPDs at (a) $2700 \mathrm{~K}$, (b) $4000 \mathrm{~K}$, and (c) $6500 \mathrm{~K}$ 